\title{
Video Compression based on Fractal Isosceles Triangular Partition using Hybrid Swarm Intelligence
}

\author{
Shraddha Pandit \\ Research Scholar \\ Department of Computer Science \\ \& Engineering \\ University Institute of Technology \\ Rajiv Gandhi Proudyogiki \\ Vishwavidyalaya, Bhopal, India
}

\author{
Piyush Kumar Shukla \\ Assistant Professor \\ Department of Computer Science \\ \& Engineering \\ University Institute of Technology \\ Rajiv Gandhi Proudyogiki \\ Vishwavidyalaya, Bhopal, India
}

\author{
Akhilesh Tiwari \\ Professor \\ Department of Computer Science \\ \& Engineering and Information \\ Technology, \\ Madhav Institute of Technology \& \\ Science \\ Gwalior, India
}

\begin{abstract}
Symmetry property of fractal transforms enriched the compression techniques of digital multi-media data. The major part of multi-media data is video and image. The diverse nature of video required more memory and bandwidth for storage and transmission. For the efficient processing of video needs compression. Isosceles triangular partition (ITP) techniques provide the symmetry of range block and domain blocks in terms of triangular shape not in a rectangle. The triangular shape reduces the process of non-overlapping of blocks and increases quality of video compression in terms of PSNR. Instead of quality improvement of video suffered from the process of compression ratio and process of encoding time of video. The bottleneck problem of isosceles triangular partition is search space and mapping of blocks. For the betterment of search space and mapping of blocks used hybrid swarm intelligence. The hybrid swarm intelligence reduces the search space and increases the efficiency of mapping and increases the compression ratio of video compression. The design algorithms simulated in MATLAB software and used some short duration of video clip and measure some standard parameters such as PSNR, encoding time, compression ratio and MSE. The design algorithm gives better results instead of isosceles triangular partition.
\end{abstract}

\section{Keywords}

Video Compression, Encoding, Isosceles Triangular Partition, Hybrid Swarm Intelligence

\section{INTRODUCTION}

Swarm intelligence enhances the coding and decoding efficiency of video compression. The process of swarm intelligence removes the bottleneck problem of domain mapping and reduces the search space for fractal transform non-overlapping blocks. The mapping of non-overlapping blocks is very slow process and increases the time of video encoding and the process of video encoding suffered from the problem of efficiency and constraints of parameters [31-33]. The swarm intelligence gives the variety of algorithms for the purpose of searching of blocks coefficient and mapping of domain blocks $[2,3]$. In this paper used two swarm algorithm one is ant colony optimization and other is particle swarm optimization [4, 14]. Both swarm algorithms are meta- heuristic and memory based iterative algorithms. The metaheuristic nature of algorithm gives the diversity for the processing of encoding and retains the quality of compressed video. The design algorithm used ant colony optimization for the processing of the group of frames for the range and domain selection. Instead of ant colony optimization particle swarm optimization used for the processing of mapping and encoding the block coefficient to domain and range. The both swarm algorithms makes hybrid swarm algorithm for the mapping and searching the reference and frame correlation for the compression. The hybrid swarm algorithm is memory based its take more iteration time and increases the complexity of process. The hybrid swarm intelligence algorithm used with the combination of partition techniques of video [8-13]. For the partition of blocks used isosceles triangular partition techniques. The triangular partition techniques generate the blocks in terms of triangle not in from of rectangle and increase the more symmetry for fractal video processing $[26,27]$. The rectangle block directly mapped the symmetry property and reduces the overlapping and improved the performance of video encoding. The isosceles triangular partition process matches the range and domain in terms of angle mapping of triangle [15]. The rest of the paper discuss isosceles triangular partition in section II. In section III discuss the process of hybrid swarm intelligence algorithm. In section IV describe the processing of compression, in section $\mathrm{V}$ describe simulation results and finally discuss conclusion and future work in section VI.

\section{ISOSCELES TRIANGULAR PARTITION}

The fractal video compression is a lossy compression strategy [31-33]. Isosceles triangular partition is a fractal technique for image compression. The isosceles triangular partition techniques enhanced the performance of encoding process of fractal video compression [5-7]. The isosceles triangular partition process used the grayscale image and video for the mapping of symmetry. The mapping of symmetry reduces the space of non-overlapping blocks of domain and range. The process of fractal encoding describes here with the figure 1[16-23]. 


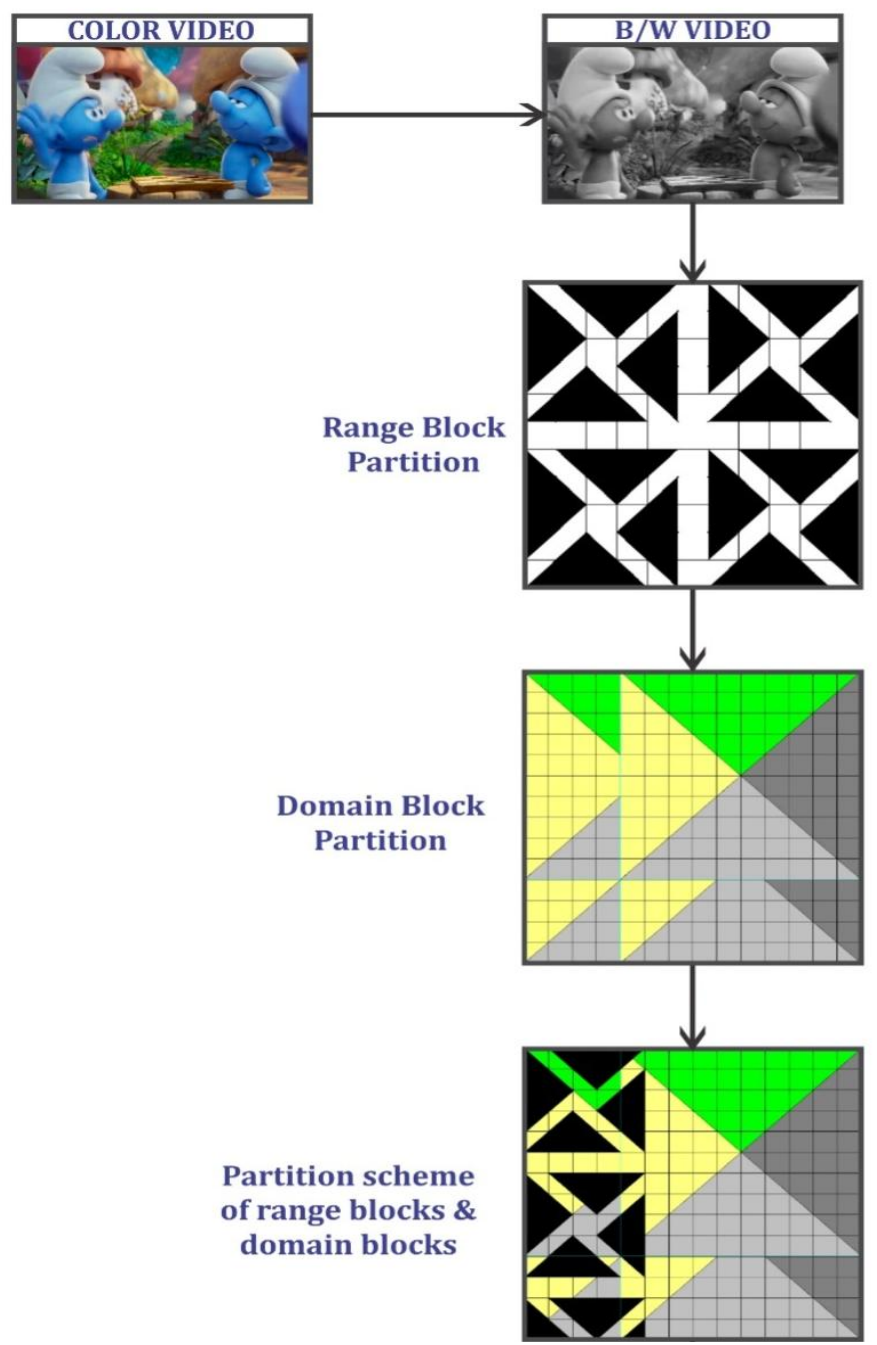

Figure 1: Process block diagram of isosceles triangular partition of RGB video

The color video RGB mode converted into grayscale video and the process of partition describe as following derivation [28].

The mapping of triangular block in concern of M1, $\mathrm{M} 2 . . . . . . . . . ., \mathrm{Mn}$ for the fractal encoding process as

$$
\begin{aligned}
& F c=R c\left(M_{1}, M_{2}, \ldots . M_{c n}\right)^{n} \\
& \text { map } \\
& =\sum_{l, p \rightarrow l} \operatorname{sim}(R(x, y, z))+D \sum_{l, \rightarrow l}^{k \rightarrow l}[S]^{k} \\
& +\sum_{, p \rightarrow l u} \text { Encode }(M(R))^{n}
\end{aligned}
$$

where $D$ and $R$ are the two range and domain blocks of triangle formation. $M_{n}$ is the last block set of fractals transform form of video encoding [29,30].

\section{HYBRID SWARM INTELLIGENCE}

The hybrid swarm algorithm is a combination of ant colony optimization and particle swarm optimization (called PHI). The combination of the swarm algorithm also called fusion of the swarm algorithm. Here ant colony algorithm decides the selection of domain with respective reference frame for the mapping of range. The process of searching is done by the particle swarm optimization [31-33]. The particle swarm optimization iterates the encoding process of fractal transform. The frames of video treat as artificial ants and the difference of frames treat as pheromone of ants and the process of ants describes here. A1 is the process of ants, $y$ is the similar point of blocks $\mathrm{S}$ is set of blocks $\mathrm{Q}$ is pheromone updates. For the processing of ants in video encoding [24, 25]

$$
\begin{array}{r}
\text { Input: GOF }=\left\{\left(\mathrm{A}_{1}, y_{1}\right),\left(\mathrm{A}_{2,} y_{2}\right), \ldots \ldots\left(\mathrm{A}_{L}, y_{L}\right)\right\} \\
S^{b}=\left\{\left(\mathrm{A}_{l_{b}}, y_{l_{b}}\right)\right\}_{l=1}^{L}, b=\{1, \ldots \ldots Q\} \\
T=\left\{\mathrm{A}_{1}, \mathrm{~A}_{2,} \ldots \ldots \ldots \mathrm{A}_{U}\right\} \\
T^{b}=\left\{\left(\mathrm{A}_{u_{b}}\right)\right\}_{u=1}^{U}, b=\{1, \ldots Q\}
\end{array}
$$

Output: $y_{u}, P u \in T / /$ here $\mathrm{T}$ is set of blocks

For each block, $b=1$ to $Q$ do

Define $S_{0}^{b}=S^{b}, T_{0}^{b}=\emptyset, U_{0}^{b}=T^{b}, M_{0 b}^{c}=I$

For iteration, $\mathrm{k}=1$ to $\mathrm{K}$ do

With $\theta_{u_{b} p}^{c} \in\left(S_{K}^{b}+T_{k}^{b}\right), P p \in\left\{1, \ldots, P_{u_{b}}\right\}$

Estimate $P l_{b} \in S_{k}^{b}, I 2 C\left(\mathrm{~A}_{l_{b}}, c\right)$

With $\quad \theta_{l_{b} p}^{c} \in\left(S_{k}^{b}+T_{k}^{b}-l\right), P_{p} \in$

$\left\{1, \ldots, P_{l_{b}}\right\}$ 
Update $S_{k+1}^{b}, T_{k+1}^{b}, U_{k+1}^{b}$

And finally proceed the optimized frames data for the processing of encoding in ant process.

Input: A set of ants $A_{n i}=\left\{a_{1}, a_{2}, \ldots, a_{j}\right\}$, and each ants $a_{i}$ with the symmetry blocks $c_{i}$ and the moves $d_{i}$, the velocity move position of blocks $C$, and the constant rate of velocity for blocks update.

Output: the SF (similar frames)

Distribute ants according to their difference

Set $D_{n i}$ as the minimal velocity in $A_{n i}$.

while $A_{n i}$ is empty

update the velocity and position of ants $A_{n i}$ to $A_{n i}^{\prime}$

Set the $S$ as the maximum frames

while $\sum_{\forall_{i} \in A^{\prime} n i}\left\lceil\frac{d_{i}}{c}\right\rceil\left\lceil\frac{s}{p c_{i}}\right\rceil\left\lfloor\frac{s}{D_{n i}}\right\rceil X c$ do

end while

end while

Return $S F$

\section{ENCODING PROCESS}

Input: A set of $\mathrm{SFs}=\left\{s f_{1}, s f_{2}, \ldots, n s f_{n}\right\}$, each symmetry frames of hybrid algorithm. the process of algorithm describes here

if the Encoding frame $n_{i}$ is added into the FV (fractal video) for each $n_{i}$ in $S F$ do

measure difference $D_{n_{i}}$ by $M A P(I T P)$

$d_{n_{i}}=D_{n_{i}}$

end for

$$
a_{n_{i}}=0
$$

measure reference frames by $I P T(f s)$

Set $t$ as encoding iteration for video end if

Find the frames $n_{j}$ with $a_{n_{j}} \geq t$ and the shortest blocks $d_{n_{j}}$ in $s f$

Find the frames $n_{k}$ with the same $d_{n_{k}}$ in $s f$

if $j \neq k$ then

$$
\begin{gathered}
t=a s f_{n_{k}} \\
j=k
\end{gathered}
$$

end if

end if

encode fractal video

$$
\begin{aligned}
s f_{n_{j}} & =f s_{n_{j}}+D_{n_{j}} \\
d_{n_{j}} & =d_{n_{j}}+D_{n_{j}} \\
t & =t+d n j
\end{aligned}
$$

end while

\section{EXPERIMENTAL ANALYSIS}

For the evaluation of the performance used some standard parameters such as PSNR, MSE, compression ratio (CR) and encoding time of video. The value of PSNR shows that the information of the quality of video. The value of compression ratio shows that the value of fast encoding process of video. All process describes here.

\section{DESCRIPTION OF DATASET}

Table1: Shows description of dataset used for compression of varied videos

\begin{tabular}{|l|l|l|}
\hline Sr.No. & \multicolumn{1}{|c|}{ Video Name } & Format of video \\
\hline 1 & Amitabh video & AVI \\
\hline 2 & Man video & AVI \\
\hline 3 & Snake video & AVI \\
\hline 4 & Cinema video & AVI \\
\hline 5 & Magic video & AVI \\
\hline 6 & Life video & AVI \\
\hline 7 & Water video & AVI \\
\hline
\end{tabular}

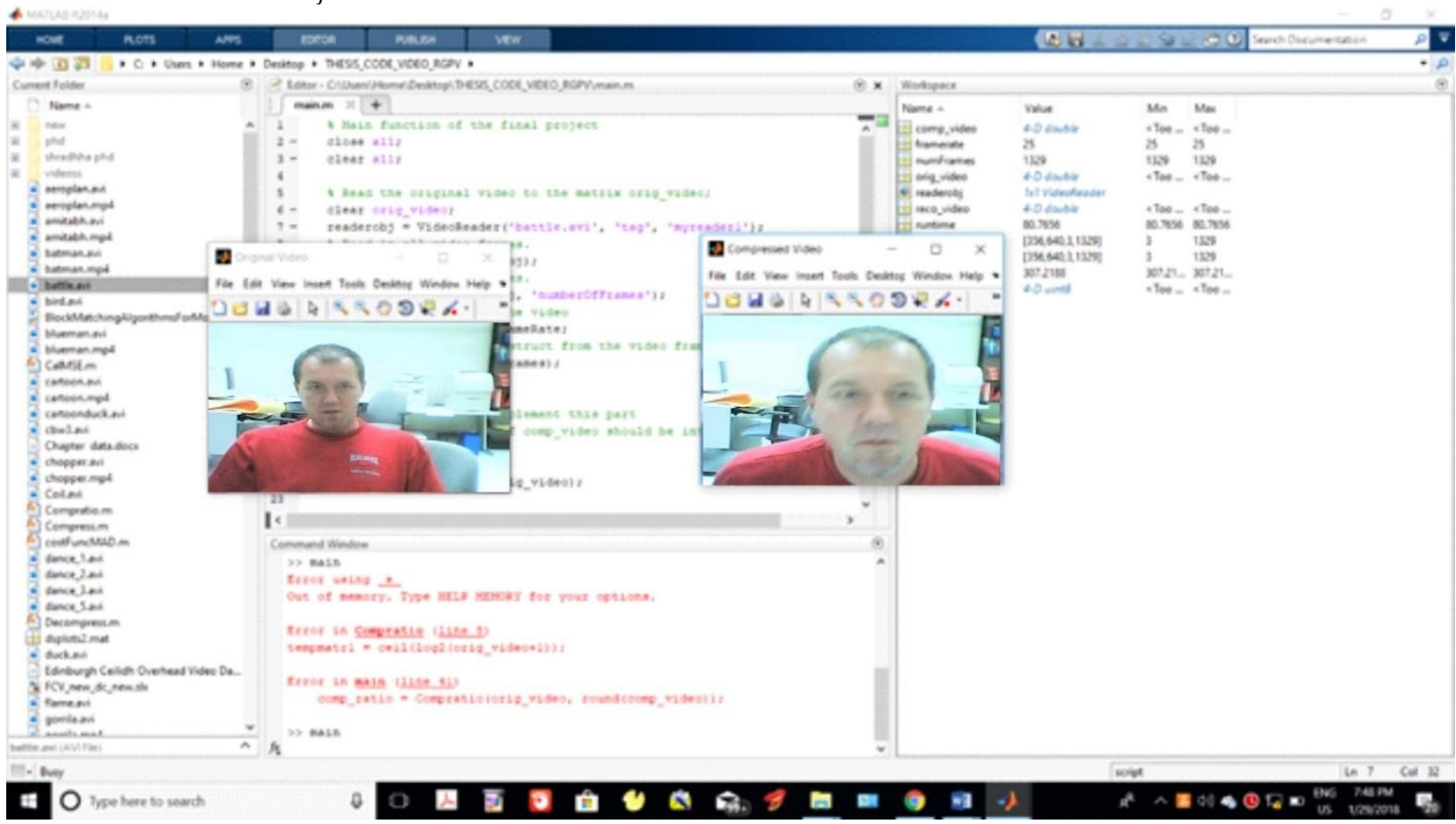

Figure 2: Shows that the original video and compressed video view of Man.avi video using ITP method. 


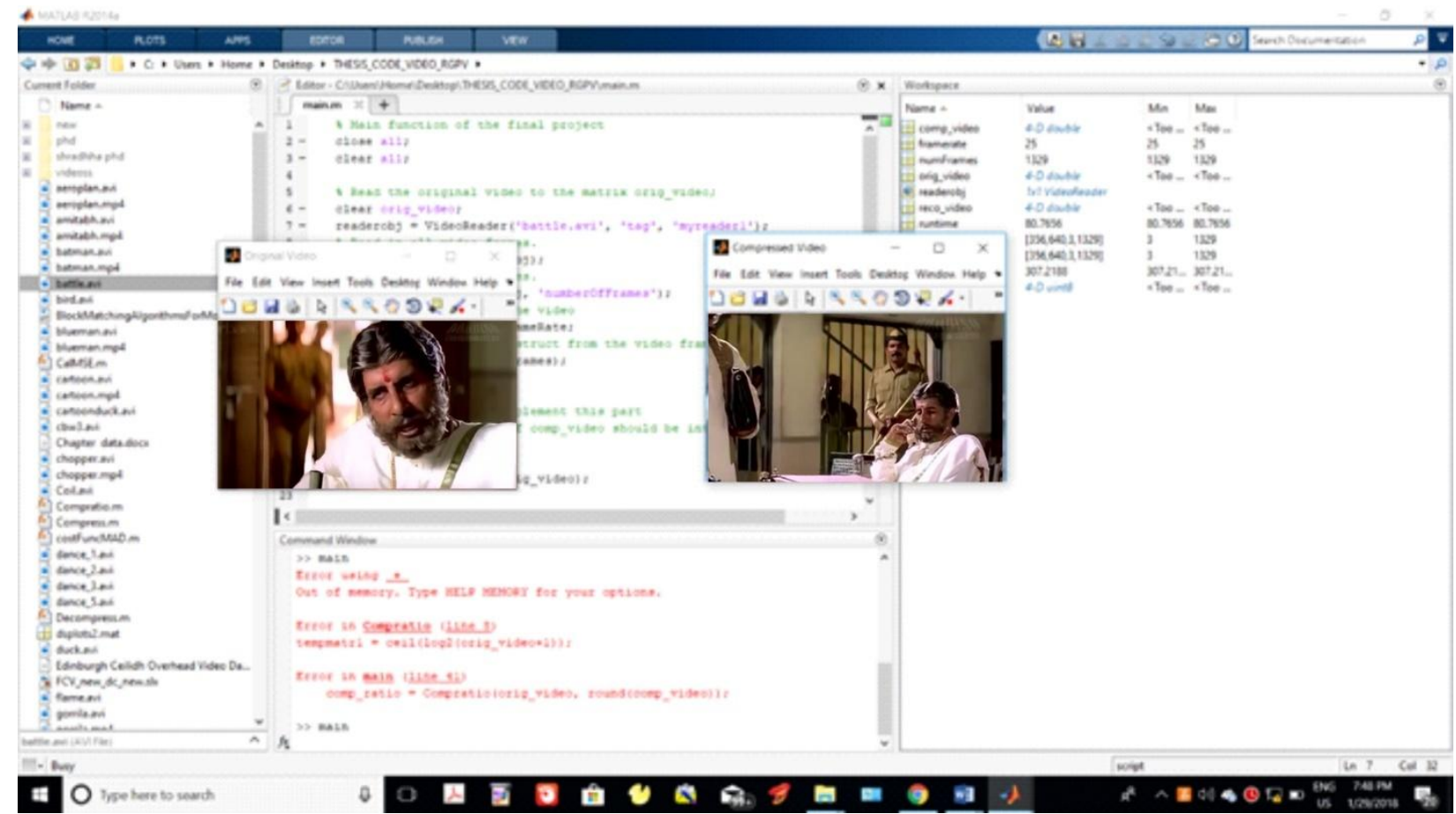

Figure 3: Shows that the original video and compressed video view of Amitabh.avi video using PHI method.

Also get the result of compression of PSNR, Compression Ratio, Mean Square Error and Encoding time for all the tested videos.

The following table shows the comparison of ITP and PHI Method with respect to Compression Ratio, Mean Square Error, Peak Signal to Noise Ratio and Encoding Time of varied AVI videos.

Table 2: Shows the Comparison of ITP and PHI Method for Man.avi video

\begin{tabular}{|l|l|l|}
\hline & ITP & PHI \\
\hline Compression Ratio & 0.78 & 0.87 \\
\hline MSE & 12.35 & 11.19 \\
\hline PSNR & 24.04 & 27.12 \\
\hline Encoding Time & 1.78 & 2.08 \\
\hline
\end{tabular}

Table 3: Shows the Comparison of ITP and PHI Method for Amitabh.avi video

\begin{tabular}{|l|l|l|}
\multicolumn{2}{c}{ for Amitabh.avi video } \\
\hline & ITP & PHI \\
\hline Compression Ratio & 0.65 & 0.91 \\
\hline MSE & 14.27 & 13.22 \\
\hline PSNR & 22.78 & 23.04 \\
\hline Encoding Time & 0.77 & 1.24 \\
\hline
\end{tabular}

Table 4: Shows the Comparison of ITP and PHI Method

\begin{tabular}{|l|l|l|}
\multicolumn{2}{c}{ for snake.avi video } \\
\hline & ITP & PHI \\
\hline Compression Ratio & 0.81 & 0.93 \\
\hline MSE & 19.12 & 18.74 \\
\hline PSNR & 19.48 & 20.34 \\
\hline Encoding Time & 0.56 & 0.89 \\
\hline
\end{tabular}

Table 5: Shows the Comparison of ITP and PHI Method for Cinema.avi video.

\begin{tabular}{|l|l|l|}
\hline & ITP & PHI \\
\hline Compression Ratio & 0.76 & 0.88 \\
\hline
\end{tabular}

\begin{tabular}{|l|l|l|}
\hline MSE & 13.45 & 12.18 \\
\hline PSNR & 25.14 & 27.22 \\
\hline Encoding Time & 1.66 & 2.16 \\
\hline
\end{tabular}

Table 6: Shows the Comparison of ITP and PHI Method for Magic.avi video.

\begin{tabular}{|l|l|l|}
\multicolumn{1}{c}{ for Magic.avi video. } \\
\hline Compression Ratio & ITP & PHI \\
\hline MSE & 0.51 & 0.72 \\
\hline PSNR & 15.01 & 13.92 \\
\hline Encoding Time & 21.98 & 23.84 \\
\hline
\end{tabular}

Table 7: Shows the Comparison of ITP and PHI Method for Life.avi video.

\begin{tabular}{|c|c|c|}
\hline & ITP & PHI \\
\hline Compression Ratio & 0.59 & 0.94 \\
\hline MSE & 18.99 & 17.54 \\
\hline PSNR & 19.99 & 20.51 \\
\hline Encoding Time & 0.96 & 1.12 \\
\hline
\end{tabular}




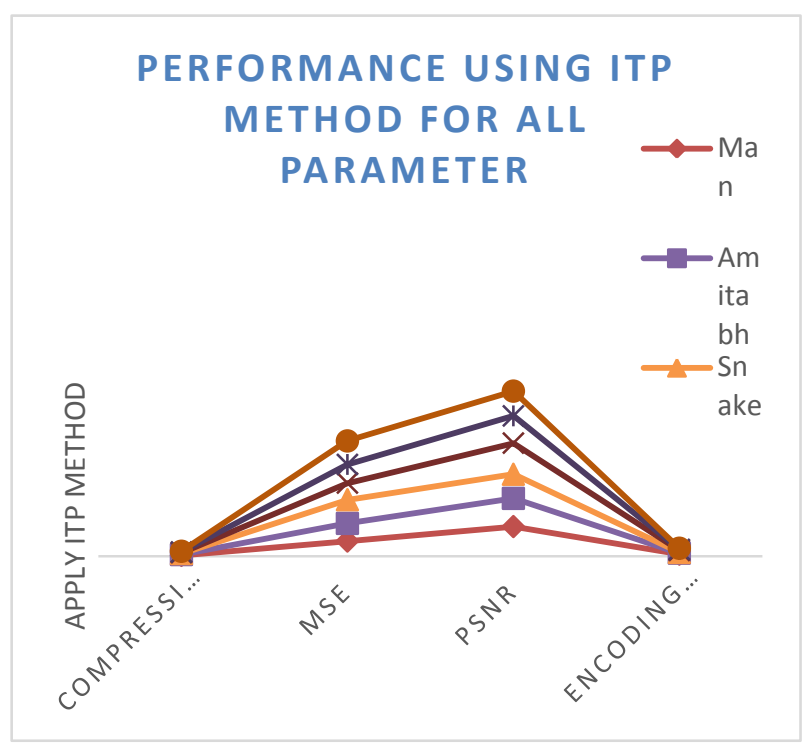

Figure 4: Shows the performance evaluation using ITPMethod for compression ratio, MSE, PSNR and Encoding Time with Man, Amitabh, Snake, Cinema, Magic and Life video avi files.

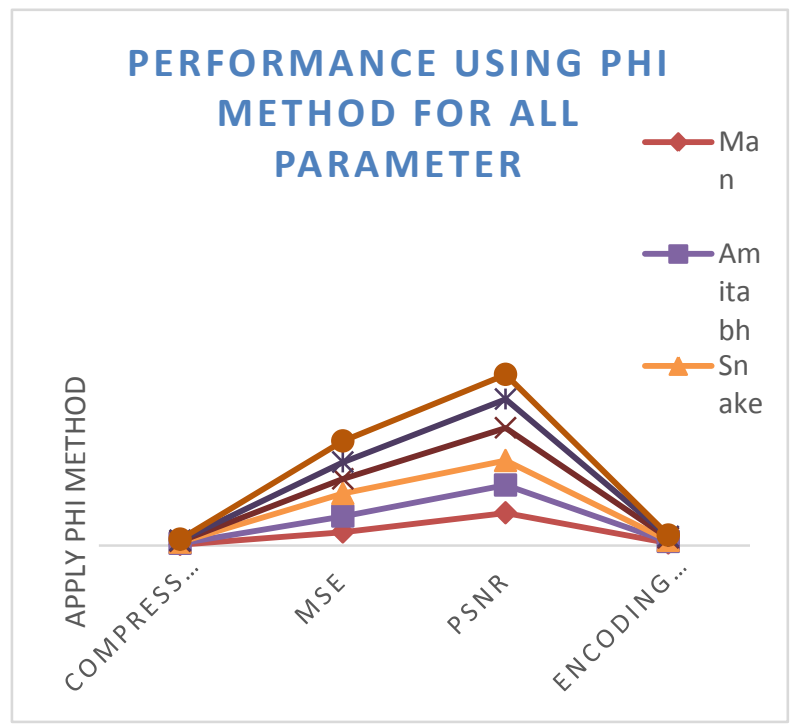

Figure 5: Shows the performance evaluation using PHIMethod for Compression Ratio, MSE, PSNR and Encoding Time with Man, Amitabh, Snake, Cinema, Magic and Life avi video files.

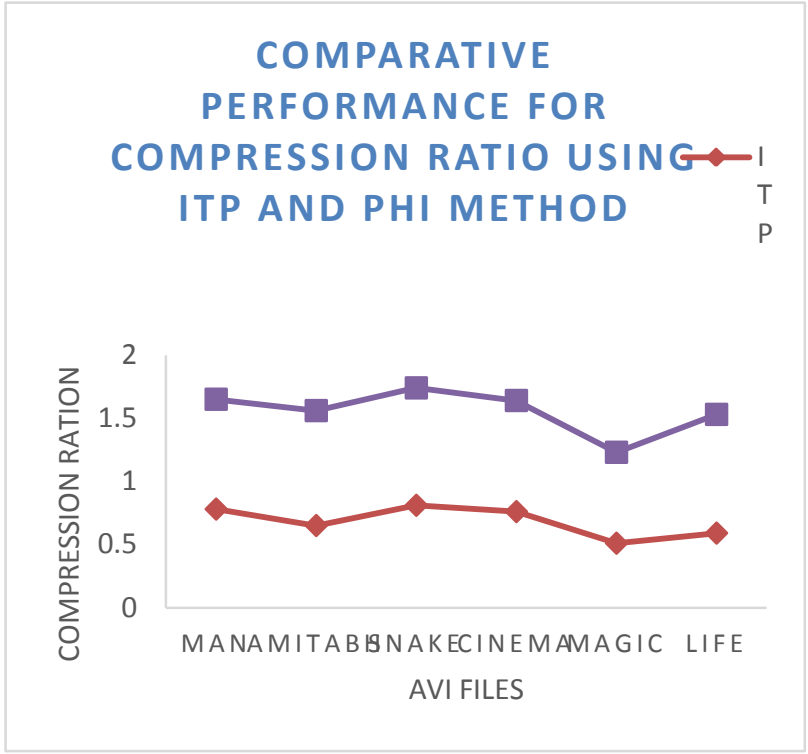

Figure 6: Shows the comparative performance of Compression Ratio using ITP and PHI method for Man, Amitabh, Snake, Cinema, Magic and Life avi video files.

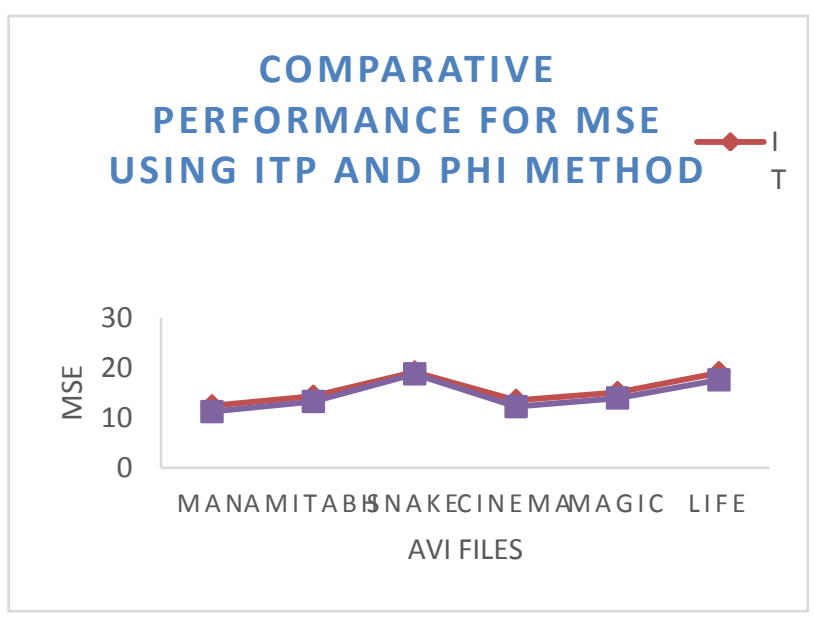

Figure 7: Shows the comparative performance of MSE using ITP and PHI method for Man, Amitabh, Snake, Cinema, Magic and Life video avi files.
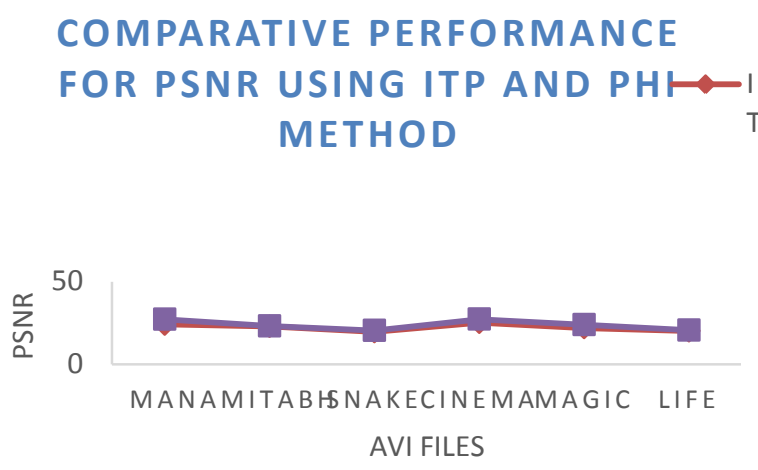

Figure 8: Shows the comparative performance of PSNR using ITP and PHI method for Man, Amitabh, Snake, Cinema, Magic and Life avi video files. 


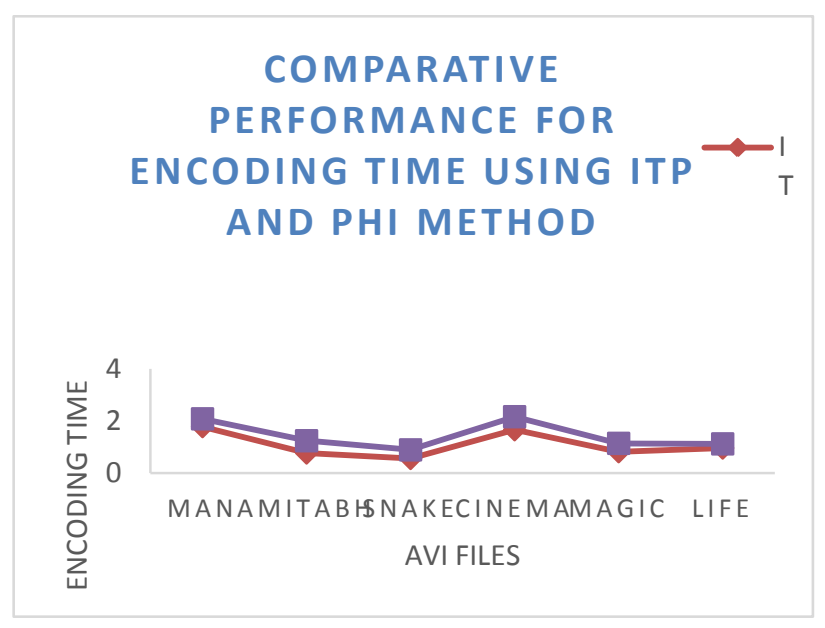

Figure 9: Shows the comparative performance of Encoding Time using ITP and PHI method for Man, Amitabh, Snake, Cinema, Magic and Life avi video files.

\section{CONCLUSION \& FUTURE WORK}

Hybrid swarm intelligence algorithm removes the bottleneck of isosceles triangular partition encoding technique in fractal video compression. The hybrid swarm algorithm reduces the searching space from the reference blocks of frames with domain mapping blocks. The enhanced mapping blocks speed up the encoding process of video compression. The hybrid algorithm plays a role of selection of reference frames and encoding frames for the processing of video. The selection of frames in terms of the group of frames for the processing of encoding. The value of MSE is also reduced and increases the value of quality of video in terms of peak signal to noise ratio. It, Furthermore, reduces the encoding time of video and the processing of fractal video compression. The overall hybrid algorithm enhances the performance of video compression in the range of $5-10 \%$. In future, we will use the video decoding process and reduce the losses of frames during the decompression of video. The frames loss occurred.

\section{REFERENCES}

[1] Yuli Zhao, Zhu and Hai Yu, "Fractal Color Image Coding Based on Isosceles Triangle Segmentation," 2010, International Workshop on Chaos-Fractal Theory and its Applications, 2010, pp. 486-490.

[2] JayavrindaVrindavanam, Saravanan Chandran Gautam K. Mahanti "A Survey of Image Compression Methods," International Conference \& Workshop on Recent Trends in Technology, 2012, Pp 12-17.

[3] Sonali V. Prof.Prachi Sorte, "An Efficient and Secure Fractal Image and Video Compression," International Journal of Innovative Research in Computer and Communication Engineering, vol.4, no.12, 2016, pp.20643-20648.

[4] Shiping Zhu, Shiping Juqiang Chen and KamelBelloulata, "An Efficient Fractal Video Sequences Codec with Multiviews", Hindawi Publishing Multiviews", 2013, pp. 1-9.

[5] Vitor de Lima, William Robson Schwartz and Helio Pedrini, "3D Searchless Fractal Video Searchless at Low Bit Rates," Journal of Mathematical Imaging and Vision, vol.45, no3, 2013, pp. 239-250.

[6] Umesh B Kodgule, B.A.Sonkamble, "Discrete Wavelet transforms based Fractal Image Compression using Parallel Approach", International Journal Approach", Computer Applications,vol.122,no.16,2015, pp.18-22.

[7] D.Venkatasekhar, P.Aruna and B.Parthiban "Fast Search Strategies using Optimization for Fractal Image Compression", International Journal Compression", Computer and Information Technology, 2013, pp. 437441

[8] Nevart A. Minas and Faten H. Mohammed Sediq, "Compression of an AVI Video File Using Fractal System", International Journal System", Computer Science Issues, vol.10, issue 5, no.2, 2013, pp. 182-189.

[9] Y. Sanchez, T. Schier, C. Hellge, T. Wiegand and D. Hong, "Efficient HTTP-based streaming using Scalable Video Coding", Signal Processing: Coding", Communication, vol.27, 2012, pp. 329-342.

[10] Lei Yu, Houqiang Li and Weiping Li, "Wireless Scalable Video Coding Using a Hybrid Digital-Analog Scheme," IEEE Transaction on Circuits System and Video Technology, vol. 24, no. 2, 2014, pp. 331-345

[11] Tariq A. Shahrul N. Fadil, Shahrul Badlishah Yaakob "A Badlishah Chaos and Neural Network Cipher Encryption Algorithm for Compressed Video Signal Transmission Over Wireless Channel," 2nd International Conference on Electronic Design, 2012, pp. 64-68.

[12] Alexandre Zaghetto and Ricardo L. de Queiroz,"Scanned Document Compression Using Block-Based Hybrid Video Codec", IEEE Transactions on Image Processing , vol.22, issue 6,2013, pp. 2420-2428.

[13] R. Sudhakar and S. Letitia, "Motion Estimation Scheme for Video Coding Using Hybrid Discrete Cosine Transform and Modified Transform Multi Hexagon-Grid Search Algorithm", Middle-East Journal Algorithm," Scientific Research, vol.23, 2015, pp. 848-855.

[14] KamelBelloulata, Amina and Shiping Zhu, "Objectbased Shiping video compression using fractals and shape-adaptive DCT," International Journal of Electronics and Communication, 2014, pp. 687-697.

[15] Shailesh D. Kamble, Nileshsingh V. Kamble, Nileshsingh Preeti Thakur Bajaj, Preeti Three-Step Bajaj, Block Matching Motion Estimation and Weighted Finite Automata based Fractal Video Compression," International Journal of Interactive Multimedia and Artificial Intelligence, 2017, pp. 27-39.

[16] S. NirmalRaj, "SPIHT: A Set Partitioning in Hierarchical Trees Algorithm for Image Compression", Contemporary Engineering Sciences, vol.8, no.6, 2015, pp. $263-270$.

[17] Shiping Zhu and Ling Zhang, "A Novel High Efficiency Fractal Multiview Video Codec", Mathematical Problems Codec", Engineering, 2015, pp. 1-12.

[18] Thomas Schier, Miska M. Hannuksela, Ye-Kui Wang and Stephan Wenger,"System Layer Integration of High Efficiency Video Coding", IEEE Transaction Coding", Circuits and Systems for Video Technology, vol. 22, no. 12, 2012, pp. 1871-1884.

[19] Rakhi Ashok Aswani Rakhi Shailesh Aswani "An Efficient D.Kamble, for Fractal Video Compression using Block Matching Motion Estimation", International Journal of Engineering Research and Applications, 

2014
pp. $25-28$

[20] R. E. Chaudhari and S. B. Dhok, "Fast Quadtree Based Normalized Cross Correlation Method for Fractal Video Compression using FFT", Journal of FFT", Engineering and Technology, vol.11, no.2, 2016, pp. 709-718.

[21] Zhehuang Huang, "Frame-groups based fractal video compression and its parallel implementation in Hadoop cloud computing environment", Multidimensional Systems environment", Signal Processing, 2017, pp. 118.

[22] Vinisha Assudani “A Novel Search Method for Fractal Video Compression using Block Matching Motion Estimation", International Journal Estimation", Computer Applications, 2016, pp. 21-25.

[23] More Menka and Menka Dayanand "Parallel Hybrid Fractal Video Coding Technique", International Journal Technique", Advanced Research in Computer Science and Software Engineering, 2014, pp. 659-663.

[24] Mohd Noor and NorulU'yuun, "Multi-view with NorulU'yuun, depth video via high efficiency video coding technique", ProQuest Dissertations Publishing, 2016, pp. 1-24.

[25] NieDaocong and Hongyuan, "a fractal information hiding algorithm based on quad-tree partition," 2015, pp. 23-37.

[26] Xuxun Liu and Desi He, "Ant colony optimization with a greedy migration mechanism for node deployment in wireless sensor networks," Journal of Network and Computer Applications, vol.39, 2014, pp. 310-318.

[27] Meie Shen, Zhi-Hui Zhan, Wei-Neng Chen, Yue-Jiao Gong, Jun Zhang and Yun Li, "Bi-Velocity Discrete Particle Swarm Optimization and Its Application to Multicast Routing Problem in Communication Networks," IEEE Transaction on Industrial Electronics, vol.61, no.12, 2014, pp. 7141-7151.

[28] Pradip Kumar Tapan Shah, Sahu, Tapan and Kanchan Chattopadhyay,"Application Mapping Santanu Chattopadhyay,"Application Network-on-Chip Using Discrete Particle Swarm Optimization," IEEE Transactions on Very Large Scle Integration Systems, Scle issue.2,2014, pp. 300-312.

[29] Parham Moradi and Mehrdad Rostami ,"Integration of graph clustering with ant colony optimization for feature selection", Knowledge-Based Systems, vol.84, Issue C, 2015, pp. 144-161.

[30] Amir Saghatforoush, Monjezi, RoohollahShiraniFaradonbeh and Danial JahedArmaghani, "Combination of neural network and ant colony optimization algorithms for prediction and optimization of fly rock and back-break induced by blasting," Engineering with Computers, Springer-Verlag London, 2015, pp. 1-13.

[31] Shraddha Pandit,Piyush Kumar Shukla and Akhilesh Tiwari, " Fractal Compression of an AVI Video File using DWT and Particle Swarm Optimization",International Journal of Computer Science and Information Security,vol.16,no.1,2018, pp. 128-131.

[32] Shraddha Pandit, Piyush Kumar Shukla and Akhilesh Tiwari, "A Proficient Video Compression Method Based on DWT \& HV Partition Fractal Transform Function," International Journal of Scientific Engineering and Technology, vol.7, issue 2,2018, pp. 20-24.

[33] Shraddha Pandit, Piyush Kumar Shukla and Akhilesh Tiwari, " Enhance the Performance of Video Compression Based on Fractal H-V Partition Technique with Particle Swarm Optimization," International Journal of Computer Science and Engineering, vol.6, issue 1,2017, pp. 31-35. 